\title{
Long-Stroke Nanopositioning Stage Driven by Piezoelectric Motor
}

\author{
Yong Wang, ${ }^{1}$ Fujun Sun, ${ }^{2}$ Junhui Zhu, ${ }^{2}$ Ming Pang, ${ }^{3}$ and Changhai $\mathbf{R u}^{2,3}$ \\ ${ }^{1}$ School of Mechatronic Engineering and Automation, Shanghai University, Shanghai 200072, China \\ ${ }^{2}$ Research Center of Robotics and Microsystem and Collaborative Innovation Center of Suzhou Nano Science and Technology, \\ Soochow University, Suzhou 215021, China \\ ${ }^{3}$ College of Automation, Harbin Engineering University, Harbin 150001, China
}

Correspondence should be addressed to Changhai Ru; rchhai@gmail.com

Received 15 July 2014; Revised 14 October 2014; Accepted 5 November 2014; Published 24 November 2014

Academic Editor: Stefania Campopiano

Copyright (C) 2014 Yong Wang et al. This is an open access article distributed under the Creative Commons Attribution License, which permits unrestricted use, distribution, and reproduction in any medium, provided the original work is properly cited.

\begin{abstract}
This paper reported a biaxial nanopositioning stage single-driven by piezoelectric motor. The employed piezoelectric motor can perform two different driving modes, namely, AC drive mode to drive in long-stroke and at high-speed and DC scanning mode with the high-resolution of several nanometers, which satisfies the requirements of both long-stroke and nanoresolution. To compensate for the effects of the variable friction force and some unpredictable disturbances, a novel backward error compensation (BEC) positioning control method integrated of the two driving modes and a double closed-loop PID controller system are proposed to obtain a high-accuracy positional motion. The experiment results demonstrate that the nanopositioning stage with large travel range of $300 \mathrm{~mm} \times 300 \mathrm{~mm}$ has a fine speed characteristic and resolution is $5 \mathrm{~nm}$. In the experiments of different travels up to $15 \mathrm{~mm}$, calibrated by a commercial laser vibrometer, the positioning accuracy is proved within $55 \mathrm{~nm}$ in $x$-axis and $40 \mathrm{~nm}$ in $y$-axis with standard deviation less than $40 \mathrm{~nm}$ in $x$-axis and $30 \mathrm{~nm}$ in $y$-axis and the final position locking can be limited to $10 \mathrm{~nm}$, meeting the requirements of micromanipulation technology.
\end{abstract}

\section{Introduction}

With the development of advanced manufacturing techniques and precision engineering, which have had the result that machined, manufactured, and measured components are no longer restricted to microscale can now be fabricated and measured at submicron and even nanoscale [1-3]. There are many applications need nanopositioning stages which involve displacements of several centimeter ranges and performances at several nanometer accuracy and resolution. For example, MEMS [4,5], nano-coordinate measuring machines (Nano-CMM) [6,7], and grating ruling machines $[8,9]$ are all such applications. Meanwhile, precision positioning system with long-stroke and high-accuracy is also an urgent requirement in biomedical engineering [10-12]. The subject of this study is also to develop a long-stroke positioning stage with fine resolution performance for the high-resolution confocal microscopic imaging and spectroscopy testing technology [13].
The traditional method to realize large stroke cross-scale precision positioning is to utilize coarse and fine combined positioning mechanism [14-16]. Regarding this type of positioning mechanism, the piezoelectric transducers (PZT) due to its high-resolution are often adopted as actuators in fine positioning $[17,18]$, but their stroke is limited to several tens of micrometers only. So they are generally combined with an additional long-stroke stage (coarse positioning stage) driven by step motor or the voice coil motor, in order to accomplish high-resolution and long-stroke properties [19, 20]. However, accompanied assembly and volumetric errors are generated, and the control systems become more complicated. Therefore, it is extremely significant to design a single-driven crossscale positioning stage capable of achieving both long-stroke and precision positioning to overcome these limitations.

In this paper, a new biaxial nanopositioning stage singledriven by piezoelectric motor is proposed for the long-stroke and precision positioning. The piezoelectric motor used as the actuator is based on the principles of the ultrasonic 
motor [21]. It has the advantages of compact structure and the capability of integrating multiscale drive modes in an all-in-one actuator. Using its two kinds of drive modes: AC drive mode with a resolution of $100 \mathrm{~nm}$ and maximum speed up to $250 \mathrm{~mm} / \mathrm{s}$ to drive in long-stroke and at high-speed and DC scanning mode with the high-resolution of several nanometers to provide nanoscale motion, resulting in both long-stroke and high-resolution requirements of a precision positioning stage, is achievable without conventional twolevel drive, which are proved through experiment.

\section{Configuration and Principles of the Positioning Stage}

2.1. Structure of the Positioning Stage. A double layout structure of the $x$-axis platform fixed on the $y$-axis platform is adopted in the stage. Each axis mainly consists of actuator, guide mechanism, and displacement feedback apparatus. Figure 1 shows the configuration and main components of the proposed stage.

In order to remain at high motion accuracy, the piezoelectric motor (model HR8), which is made by Nanomotion Co. of Israel, is utilized as the actuator to drive the $x$-axis and $y$-axis platform. The piezoelectric motor is installed and fixed in the middle position of the mounting base of $x$ - or $y$-axis. The $x$-axis platform is driven by one HR8, while the $y$-axis platform is driven by two HR8 in the same side considering the load. The moving platform is supported by two pairs of THK precision cross roller guides in each axis. Due to its high-resolution ( $5 \mathrm{~nm}$ resolution), highspeed measurement, and high anti-interference ability, the Renishaw linear grating scale (model RELM430) is utilized as the precise displacement sensors, so that the position feedback of linear motion in each axis can be detected to realize the closed-loop positioning control. The travel range of the stage approached $300 \mathrm{~mm} \times 300 \mathrm{~mm}$.

2.2. Principle of Piezoelectric Motor HR8. The actuator system consists of the piezoelectric motor HR8 and a drive amplifier (model AB2, supplied by Nanomotion Co. of Israel). The two components are combined to create the piezoelectric effect, which converts an electrical field into a mechanical motion. Each HR8 motor is composed of eight same piezoelectric ceramic elements [22]. Here one of them is selected to be analyzed, as is shown in Figure 2.

When the drive voltage is applied across the A electrode and $\mathrm{C}$ electrodes (or B electrode and $\mathrm{C}$ electrode) at a resonate frequency of $39.6 \mathrm{KHz}$, the longitudinal extension and transverse bending vibration modes of the piezoelectric element are simultaneously excited [23], exactly as is modal simulated by the ANSYS FEM software and shown in Figure 2. A schematic illustration of the two vibration modes is presented in Figure 3. Their simultaneous excitation causes the ceramic fingertip pusher in the front of the motor to execute a small elliptical motion. The ceramic fingertip pusher is appropriately preloaded by the preload spring $\mathrm{K}$ and is located in contact with a ceramic driving strip mounted on the moving platform. Then this elliptical motion drives the stage by means of the friction force between the pusher and ceramic driving strip to create linear motion of the stage.

\subsection{Positioning Control Method Integrated of Two Driving} Modes. The combination of the HR8 motor and its associated $\mathrm{AB} 2$ driver can mainly perform the motion in there drive modes: AC mode, Gate mode, and DC scanning mode, which cover approximately scales of millimeter, micrometer, and nanometer, respectively [22]. Figure 4 presents the driving schematic illustration of the AC drive mode and DC scanning mode. In electronics, the piezoelectric motor can be equivalent to a capacitor.

In $\mathrm{AC}$ mode, the piezoelectric motor is driven continuously by applying a continuous sine wave driving voltage with a resonate frequency of $39.6 \mathrm{KHz}$, which creates continuous steps for long-stroke with a resolution of $100 \mathrm{~nm}$ and maximum speed up to $250 \mathrm{~mm} / \mathrm{s}$. Its driving direction can be controlled by the switch S1 and S2 while the Gate drive mode is actually a kind of intermittent AC drive mode. The last DC scanning mode is a unique mode of the AB2. Its driving principle is the same as the conventional PZT used for microdisplacement driving. The DC scanning mode makes use of the bending mode principle of the PZT and is excited by giving a DC voltage so that ceramic fingertip pusher can generate a small motion proportional to the input voltage. It is equivalent to an ordinary PZT with a driving displacement range from $-300 \mathrm{~nm}$ to $300 \mathrm{~nm}$ and highresolution of several nanometers in this mode. Therefore, with a proper combination of the AC and DC modes, the stage can perform long-stroke and fine motion to nanometer resolution.

The three driving modes of AB2 driver have been used for long-stroke precision positioning in previous reports. Typically, Fan et al. [24-26] adopt the AC mode and Gate mode to make the moving platform close to the target point within $5 \mu \mathrm{m}$ and $30 \mathrm{~nm}$, successively. And lastly in DC mode, the motor is utilized to adjust the position in several nanometers and finally to lock at the target position. In their research, the positioning error of AC mode is forward compensated successively in Gate mode and DC mode. However, complicated control systems and algorithm are required for the switch control of these three driving modes in micro-nano-scale. In this study, a novel backward error compensation (BEC) positioning control method, integrated of the AC mode and DC scanning mode, is proposed to perform long-stroke and fine motion. Figure 5 presents the control processes flow chart of this positioning control strategy. Firstly, the AC driving mode creates continuous steps for long-stroke with moderate speed to approach the desired position. The position feedback of linear motion in each axis is constantly detected by the linear grating scale. Once detected the motion of the stage has reached the desired position, a "Halt" motion command is issued immediately by the controller to control the HR8 piezoelectric motor stop driving the stage. Then the stage is locked by the hold torque produced by the compression of the ceramic fingertip pusher to the ceramic driving strip. However, due to the presence of mechanical vibration and environmental disturbance, a 


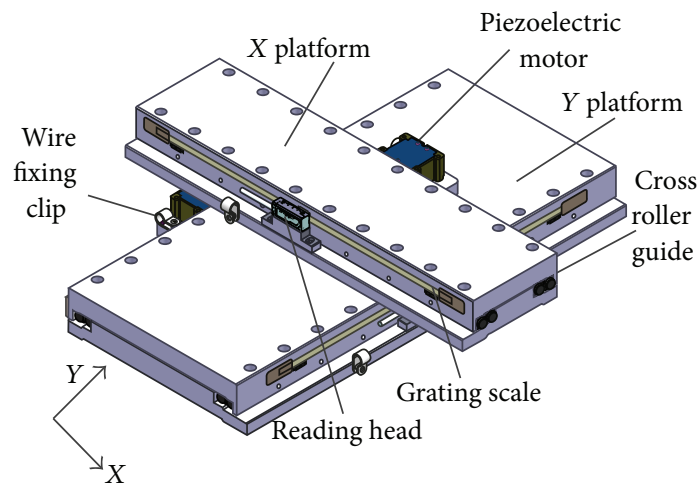

(a)

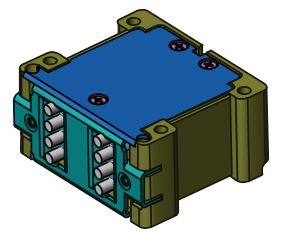

(b)

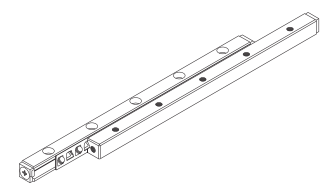

(c)

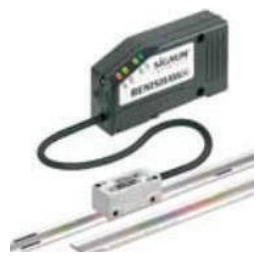

(d)

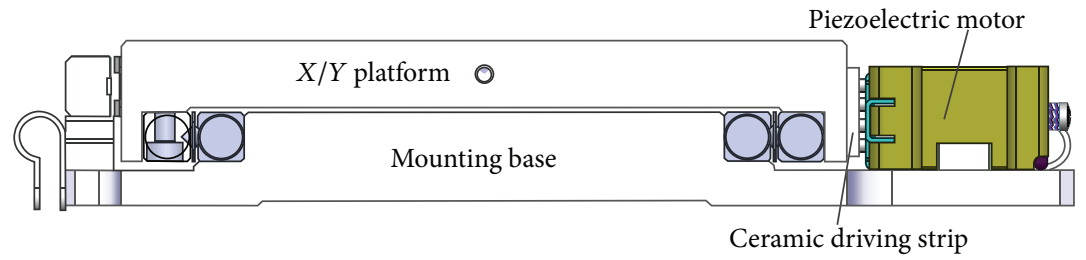

(e)

FIGURE 1: Components of the stage. (a) Structure of the whole stage; (b) diagram of the actuator; (c) diagram of the guide mechanism; (d) diagram of the displacement feedback apparatus; (e) diagram of motor installation.

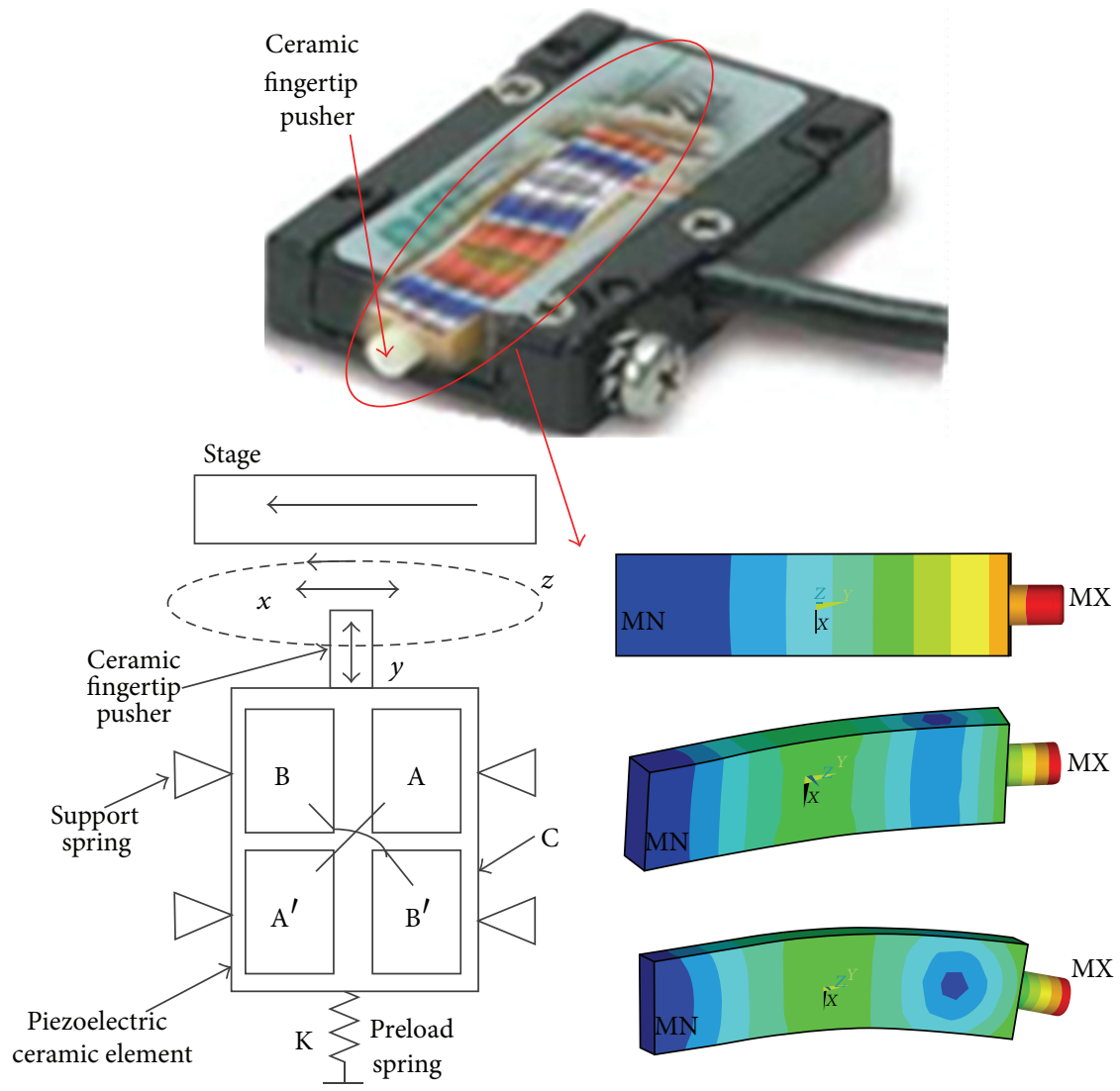

FIGURE 2: Structural diagram and schematic representation of the piezoelectric motor. 


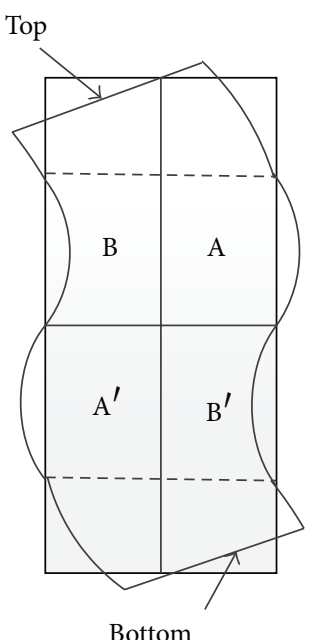

Bottom

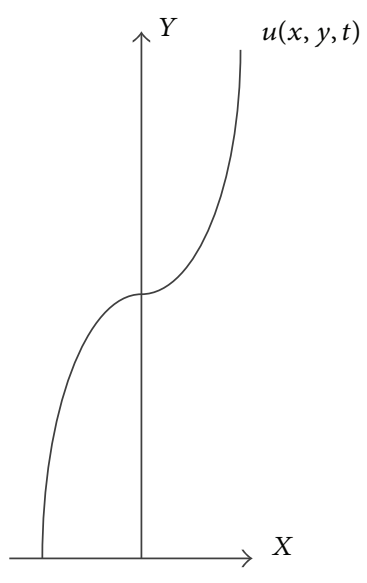

Longitudinal mode

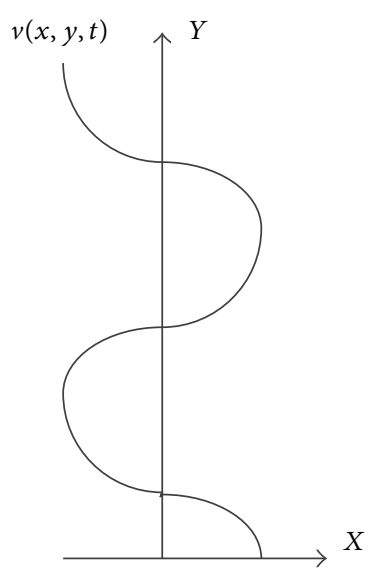

Bending mode

FIGURE 3: Longitudinal and bending vibration modes of piezoelectric motor.

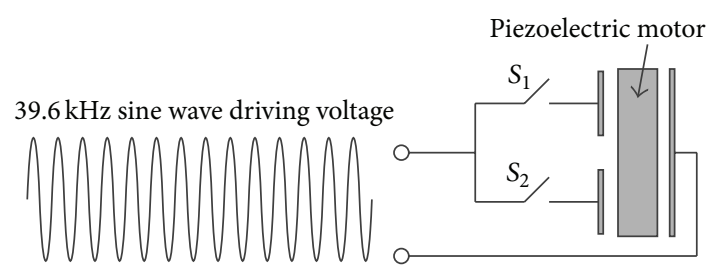

(a)

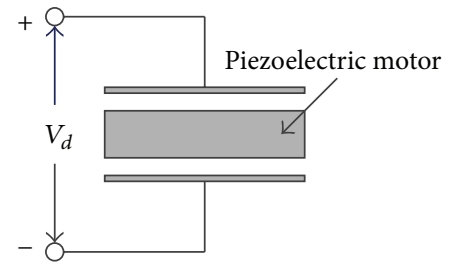

(b)

Figure 4: The driving schematic illustration. (a) AC drive mode; (b) DC scanning mode.

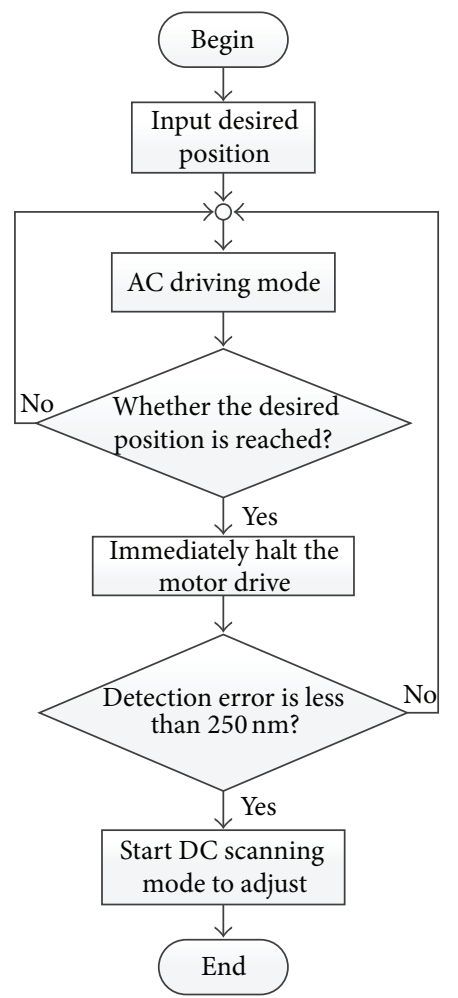

FIGURE 5: Flow chart of the BEC positioning control method. forward position error is emerged consequentially. The error compensation threshold is set as $250 \mathrm{~nm}$ to ensure that the position error is within the $300 \mathrm{~nm}$ driving range of DC scanning mode. When the position error detected by the grating scale is less than $250 \mathrm{~nm}$, the $\mathrm{HR} 8$ piezoelectric motor is controlled instantly to start the DC scanning mode to compensate the emerged position error backward. If the detection position error is more than $250 \mathrm{~nm}$, the piezoelectric motor is still controlled to drive the stage backward with AC driving mode, until the position error is detected less than $250 \mathrm{~nm}$, the DC scanning mode is switched to make the stage scan to the desired position and lock at the position.

2.4. Control System of the Nanopositioning Stage. The positioning control system for the proposed stage comprises a motion control card made by ACS Motion Control, Ltd., an actuation system (three HR8 piezoelectric motors and associated driver amplifiers AB2), a position feedback detection system (linear grating scale), a PID speed loop controller, and a PID position loop controller, as schematically shown in Figure 6. Both the speed loop controller and position loop controller are programmed in Visual $\mathrm{C}++$ Builder where the C Library is supplied by the ACS motion control, Ltd. And the control signals are interfaced via a PCI bus of the ACS control card. The position feed signals are decoded and subdivided by a Renishaw subdivision box to nanometer resolution. 
As mentioned in Section 2.3, the BEC positioning control method is divided into two motions: the long-stroke and successive motion by AC mode and position error compensation by DC scanning mode. In AC mode, the stage is required to control at a low and stable speed. However, in practice, nonconstant friction force between the moving platform and the guide way will introduce notable accelerations that will cause unsteady-state motion resulting in positioning errors. Although the positioning error of AC mode can be compensated by DC scanning mode, it is necessary to reduce the positioning error in $\mathrm{AC}$ mode because the movement range in DC scanning mode is limited to $300 \mathrm{~nm}$. Then in DC scanning mode, the HR8 works like a conventional PZT actuator to compensate the positioning error of AC mode and finally to lock at the desired position. Due to the fact that some unpredictable disturbances may affect the position accuracy, a closed-loop control scheme is thus necessary in DC scanning mode. In this study, a double closed-loop PID controller is proposed for the whole positioning control process. Figure 7 presents the principle diagram of the double closed-loop PID controller. The speed loop is as the inner loop in this double closed-loop system, for AC driving mode to drive the motor HR8 working at the desired speed, where the speed is used as the controlled parameter. The actual speed is feedback by encoder of the motor. The position loop is used for the DC scanning mode to compensate positioning error and lock position, where the displacement is used as the controlled parameter while the grating scale is always detecting the position of stage in real-time in the whole system. Only the switch threshold is detected; the AC driving mode will switch to DC scanning mode. In the proposed positioning system, the switch threshold between the AC drive mode and DC scanning mode is set as $250 \mathrm{~nm}$.

\section{Experimental Results}

To test the performance of proposed positioning stage and above control scheme, two parts of experiments are designed. Before the experiment, the double closed-loop PID parameters are adjusted to the best in the ACS control software, where the position gain $K_{P P}$ is 200 for the $x$-axis and 250 for the $y$-axis in position loop controller while in speed loop controller, the velocity gain $K_{V P}$ and the integrator gain $K_{V I}$ are 35 and 400 for the $x$-axis and 50 and 250 for the $y$-axis, respectively.

Firstly, the performance of speed control in AC drive mode and positioning control in DC scanning mode can be testified as follows.

In AC drive mode, the desired speed is $1 \mathrm{~mm} / \mathrm{s}$. The displacement feedback of linear motion in each axis is detected by the linear grating scale. Then the speed of stage could be obtained through the time interval differential of displacement. The performance of speed control by the PID speed loop controller is shown in Figure 8. The speed curves of $x$-axis and $y$-axis seem to be not smooth enough, because the sampling time interval of $50 \mathrm{~ms}$ to calculate current speed is very short so that speed variation is inevitable. However, because the grating is in noncontact configuration and its
TABLE 1: Positioning errors analysis of $x$-axis (unit in $\mathrm{nm}$ ).

\begin{tabular}{lccccccc}
\hline Travel & 1st & 2nd & 3rd & 4 th & 5 th & Mean & $\sigma$ \\
\hline $3 \mathrm{~mm}$ & -14 & 23 & 6 & 3 & 6 & 5 & 13 \\
$6 \mathrm{~mm}$ & 48 & -36 & 5 & 12 & -2 & 5 & 30 \\
$9 \mathrm{~mm}$ & 53 & 23 & -20 & 2 & -10 & 10 & 29 \\
$12 \mathrm{~mm}$ & 32 & -24 & -42 & 52 & -22 & -1 & 40 \\
$15 \mathrm{~mm}$ & 8 & -23 & -14 & -7 & 50 & 3 & 29 \\
\hline
\end{tabular}

TABLE 2: Positioning errors analysis of $y$-axis (unit in $\mathrm{nm}$ ).

\begin{tabular}{lccccccc}
\hline Travel & 1st & 2nd & 3rd & 4th & 5 th & Mean & $\sigma$ \\
\hline $3 \mathrm{~mm}$ & 3 & -26 & 5 & 6 & 15 & 1 & 16 \\
$6 \mathrm{~mm}$ & 1 & 5 & -18 & -33 & 0 & -9 & 16 \\
$9 \mathrm{~mm}$ & -23 & 11 & 30 & -27 & 15 & 1 & 25 \\
$12 \mathrm{~mm}$ & 38 & -26 & 9 & -16 & -30 & -5 & 28 \\
$15 \mathrm{~mm}$ & 10 & 12 & -36 & -30 & -29 & -15 & 24 \\
\hline
\end{tabular}

errors are corrected after normalization, this kind of speed variation will not affect the precise reading of the grating.

In DC scanning mode, the performance of displacement control by the PID position loop controller is shown in Figure 9. The results show that the positioning system driven by DC scanning mode is able to converge in $2 \mathrm{~s}$ and can lock the position for a long time with the shift within $10 \mathrm{~nm}$.

In order to test the resolution of the positioning stage, the positioning stage is driven by DC scanning mode with stepped movement of $1 \mathrm{~nm}, 5 \mathrm{~nm}$, and $10 \mathrm{~nm}$, respectively. The duration of every movement step distance is set as $20 \mathrm{~s}$. Due to the test environment disturbances, it is less obvious of the $1 \mathrm{~nm}$ step distance. Figure 10 presents the test resolution of the positioning stage. The test results show that the resolution of the stage is $5 \mathrm{~nm}$. The stage can move step by step with $5 \mathrm{~nm}$ from 0 to $100 \mathrm{~nm}$.

If the noise level of the test environment can be further reduced, the minimum resolution of the positioning stage will be further enhanced.

Secondly, to prove the precision of grating scale and estimate the performance of the whole positioning system, a commercial laser vibrometer (model OFV-5000-S, supplied by Polytec Co., Ltd.) is employed as the displacement calibrator. It can measure up to $20 \mathrm{~m} / \mathrm{s}$ vibration velocity, and its measurement frequency can reach $1.5 \mathrm{MHz}$, which can be measured from the nanometer to several meters level displacements. The readings of the laser vibrometer and grating scale are sampled simultaneously. The whole positioning system is put on a vibration isolation platform, as shown in Figure 11.

In this part of test experiment, five different travel lengths of $3 \mathrm{~mm}, 6 \mathrm{~mm}, 9 \mathrm{~mm}, 12 \mathrm{~mm}$, and $15 \mathrm{~mm}$ were carried out with the assistance of the error compensation scheme for the test environmental disturbance error. For each travel length, the positioning experiment is repeated 5 times and the test errors are listed in Tables 1 and 2 .

It is demonstrated that the proposed long-stroke nanopositioning can achieve the positioning error less than $55 \mathrm{~nm}$ in $x$-axis and $40 \mathrm{~nm}$ in $y$-axis and the standard deviation less 


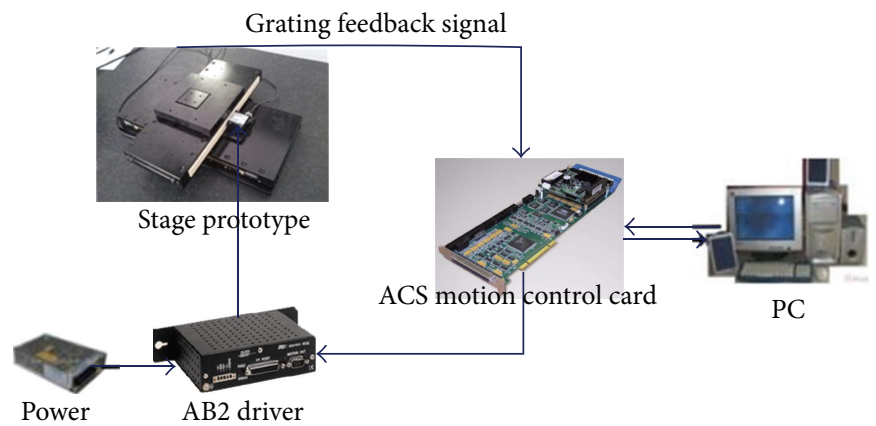

FIGURE 6: The control system structure of the stage.

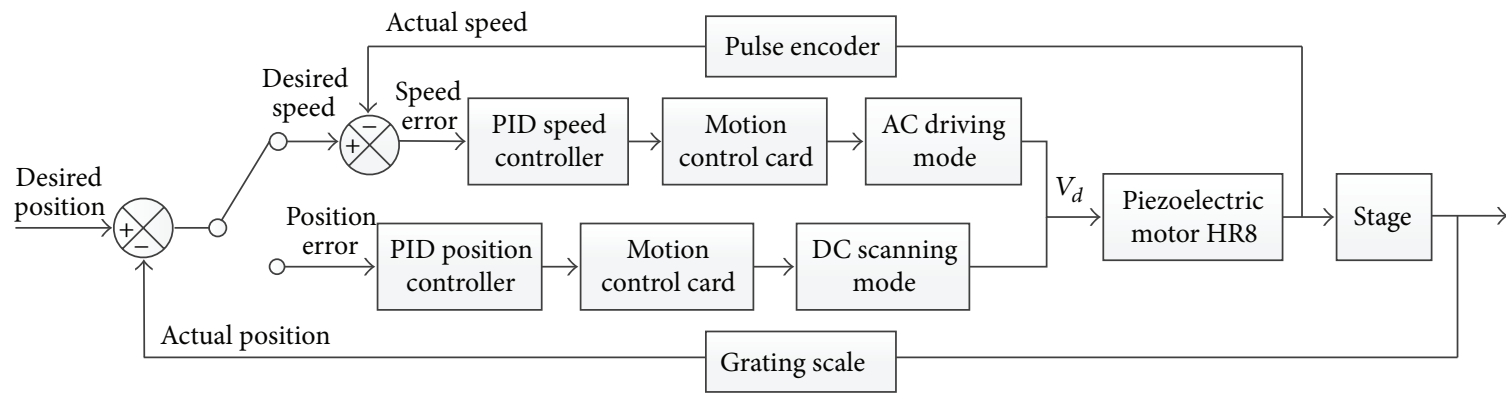

FIGURE 7: Block diagram of the double closed-loop PID controller.

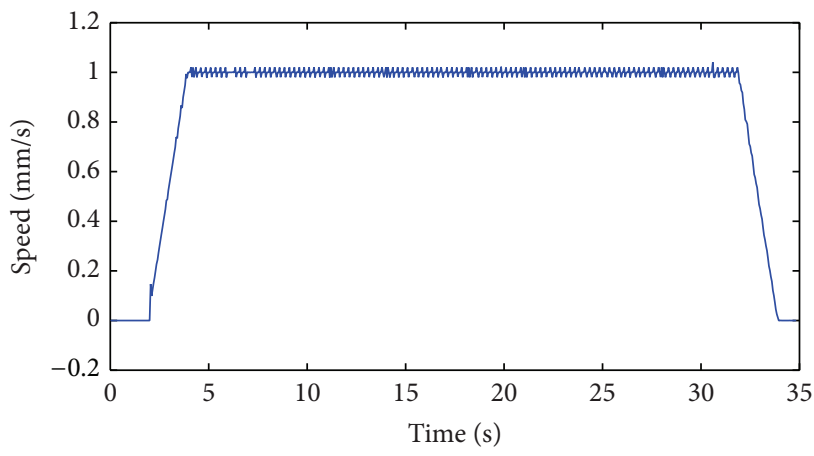

(a) $x$-axis

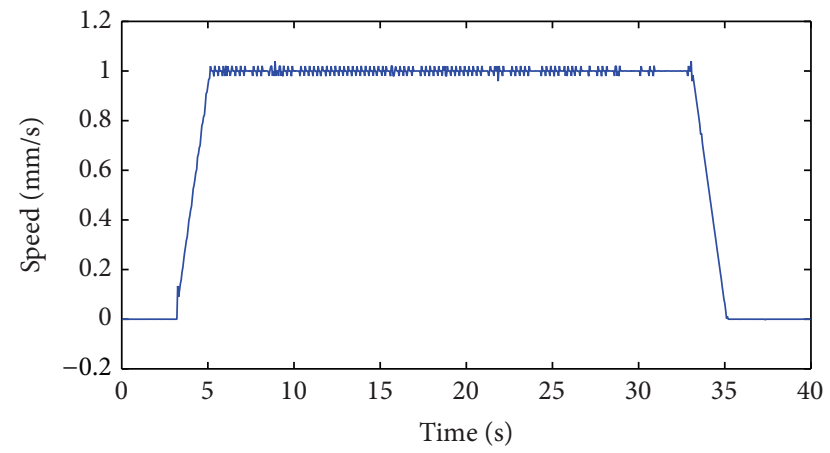

(b) $y$-axis

FIGURE 8: The speed control in AC drive mode.

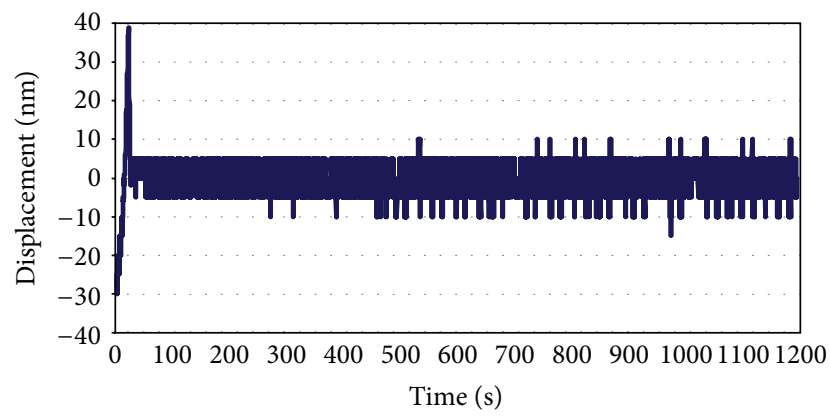

FIGURE 9: The positioning control performance in DC scanning mode. 


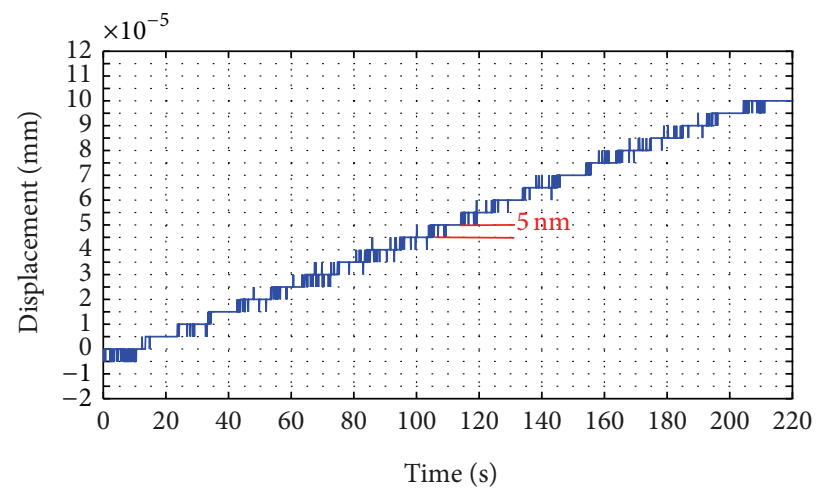

FIGURE 10: Resolution curve of the positioning stage.

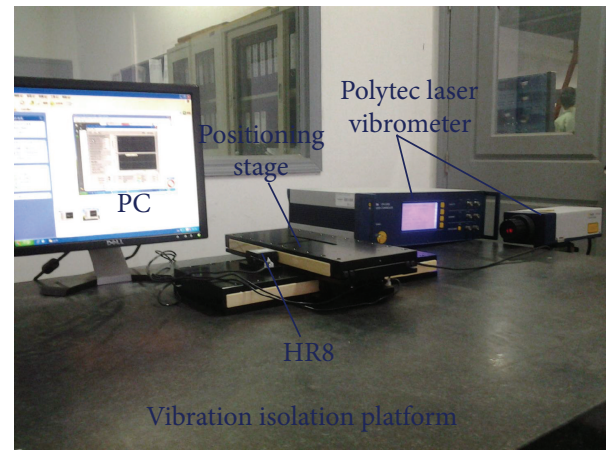

FIgURE 11: The experimental photo.

than $40 \mathrm{~nm}$ in $x$-axis and $30 \mathrm{~nm}$ in $y$-axis within the travel range of $15 \mathrm{~mm}$. The positioning performance of the $y$-axis is better than $x$-axis, because the $y$-axis of the positioning stage is more stable than the $x$-axis, which is less affected by environmental disturbance. If the whole system is put in a constant temperature chamber where the temperature is constant and the vibration and air flow are isolated effectively, the positioning accuracy will achieve better.

\section{Conclusion}

This paper has presented a new biaxial long-stroke and precision positioning stage single-driven by piezoelectric motor. Its positioning control system comprises three piezoelectric motors and associated drivers, two linear grating displacement sensors, a PID speed loop controller, and a PID position loop controller. The control system mainly supports two different driving modes, namely, the AC mode for millimeter and micrometer displacement and DC scanning mode for nanometer displacement. It makes the multiscale displacement driven by a single actuator possible. The employed grating is featured with long-stroke and nanometer resolution/accuracy. The velocity can be controlled in smooth motion by the PID speed loop controller in AC mode, and the position can be controlled at the target approaching by the PID position loop controller in DC scanning mode. Besides, a novel backward error compensation (BEC) positioning control strategy integrated of the two driving mode enhances the positioning accuracy. The experimental results have shown that the proposed stage is capable of performing both long-stroke and precise motion with fine speed characteristic and nanometer level positioning accuracy. These results demonstrated that the proposed stage has significant potential for applications such as MEMS, micromachining, and biomedical engineering.

\section{Conflict of Interests}

The authors declare that there is no conflict of interests regarding the publication of this paper.

\section{Acknowledgments}

The authors gratefully acknowledge financial support from the National High Technology Research and Development Program of China (Grant no. 2012AA040507), Instrument Development Major Program of National Natural Science of China (Grant no. 61327811), National Natural Science of China (Grant no. 61233010), and Jiangsu Natural Science Funds for Distinguished Young Scholar (Grant no. BK2012005).

\section{References}

[1] W. Gao, Springer, 2010.

[2] E. B. Brousseau, S. S. Dimov, and D. T. Pham, "Some recent advances in multi-material micro- and nano-manufacturing," The International Journal of Advanced Manufacturing Technology, vol. 47, pp. 161-180, 2010.

[3] S. Kim and W. Moon, "A micro-pull-off test machine for reliable measurement of adhesive forces on micro/Nano-scale areas," Journal of Adhesion, vol. 87, no. 2, pp. 139-153, 2011.

[4] A. G. Fowler, A. N. Laskovski, A. C. Hammond, and S. O. R. Moheimani, "A 2-DOF electrostatically actuated MEMS nanopositioner for on-chip AFM," Journal of Microelectromechanical Systems, vol. 21, no. 4, pp. 771-773, 2012.

[5] S. H. Yang, Y. S. Kim, and J. M. Yoo, "Microelectromechanical systems based Stewart platform with sub-nano resolution," Applied Physics Letters, vol. 101, no. 6, Article ID 061909, 2012. 
[6] T. Hausotte, B. Percle, and G. Jäger, "Advanced three-dimensional scan methods in the nanopositioning and nanomeasuring machine," Measurement Science and Technology, vol. 20, no. 8, Article ID 084004, 2009.

[7] C. L. Chu, C. T. Lu, H. C. Chen, J. S. Ke, and C. M. Chang, "Development of a micro-CMM with scanning touch probe and high-precision coplanar platform," in Proceedings of the 6th International Symposium on Precision Mechanical Measurements, 89161M, vol. 8916 of Proceedings of SPIE, Guiyang, China, 2013.

[8] Y. Shen, D. C. Liu, G. F. Lian, and C. A. Zhu, "Modeling and Control of Ultra Precision positioning system for a grating ruling machine," Applied Mechanics and Materials, vol. 110, p. 2647, 2012.

[9] L. T. Sun, J. S. Wang, Z. Q. Han, and C. A. Zhu, "Active vibration suppression based on intelligent control for a long-range ultraprecise positioning system," Journal of Applied Mechanics and Materials, vol. 87, pp. 123-128, 2011.

[10] C. Ru, J. Liu, M. Pang, and Y. Sun, "Controlled ultrasonic microdissection of thin tissue sections," Biomedical Microdevices, vol. 16, no. 4, pp. 567-573, 2014.

[11] M. Gauthier and E. Piat, "Control of a particular micro-macro positioning system applied to cell micromanipulation," IEEE Transactions on Automation Science and Engineering, vol. 3, no. 3, pp. 264-271, 2006.

[12] S. Hu and D. Sun, "Automatic transportation of biological cells with a robot-tweezer manipulation system," International Journal of Robotics Research, vol. 30, no. 14, pp. 1681-1694, 2011.

[13] W. Zhao, J. Guo, L. Qiu, Y. Wang, J. Meng, and D. Gao, "Low transmittance ICF capsule geometric parameters measurement using laser differential confocal technique," Optics Communications, vol. 292, pp. 62-67, 2013.

[14] W. Dong, J. Tang, and Y. ElDeeb, "Design of a linear-motion dual-stage actuation system for precision control," Smart Materials and Structures, vol. 18, no. 9, Article ID 095035, 2009.

[15] K. Kawashima, T. Arai, K. Tadano, T. Fujita, and T. Kagawa, "Development of coarse/fine dual stage using pneumatically driven bellows actuator and cylinder with air bearings," Precision Engineering, vol. 34, no. 3, pp. 526-533, 2010.

[16] C.-H. Liu, W.-Y. Jywe, Y.-R. Jeng, T.-H. Hsu, and Y.-T. Li, "Design and control of a long-traveling nano-positioning stage," Precision Engineering, vol. 34, no. 3, pp. 497-506, 2010.

[17] T. Zhang, L. N. Sun, and H. G. Cai, "Study on the fundamental characteristics of piezoelectric element," Optics and Precision Engineering, vol. 6, no. 5, pp. 26-32, 1998.

[18] L. Ma, W. Xie, B. Liu, and L. N. Sun, "Design of micropositioning stage with flexure hinge," Optics and Precision Engineering, vol. 22, pp. 338-345, 2014.

[19] D. Q. Liu, Y. M. Huang, Z. F. Wu, and Y. Yang, "Position error compensation for a Macro-micro feed system," Journal of Mechanical Science and Technology, vol. 30, p. 645, 2011.

[20] Q. S. Xu and J. Mech, "Design, testing and precision control of a novel long-stroke flexure micropositioning system," Mechanism and Machine Theory, vol. 70, pp. 209-224, 2013.

[21] C. S. Zhao, Springer, 2011.

[22] Nanomotion Ltd, “AB2 driver user manual," D/N: AB0245800000 REV: C, 2011, http://www.nanomotion.com.

[23] C. Y. Xu, Hefei University of Technology, 2008.

[24] K. C. Fan and Z. F. Lai, "An intelligent nano-positioning control system driven by an ultrasonic motor," International Journal of Precision Engineering and Manufacturing, vol. 9, no. 3, pp. 4045, 2008.
[25] F. Cheng, K. C. Fan, J. W. Miao, B. K. Li, and H. Y. Wang, "A BPNN-PID based long-stroke nanopositioning control scheme driven by ultrasonic motor," Precision Engineering, vol. 36, no. 3, pp. 485-493, 2012.

[26] H. Y. Wang, K. C. Fan, J. K. Ye, and C. H. Lin, "A longstroke nanopositioning control system of the coplanar stage," IEEE/ASME Transactions on Mechatronics, vol. 19, no. 1, pp. 348-356, 2014. 

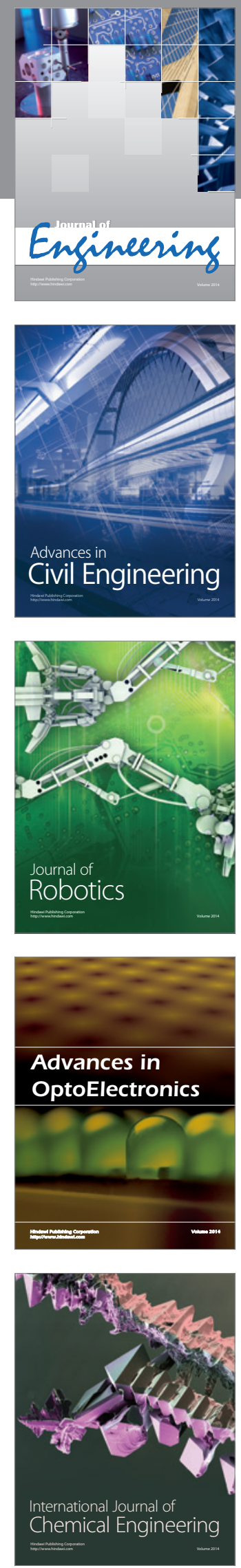

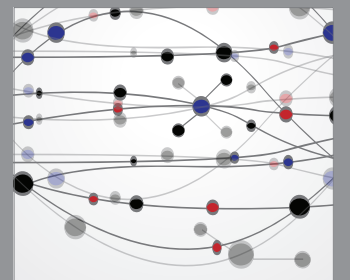

The Scientific World Journal
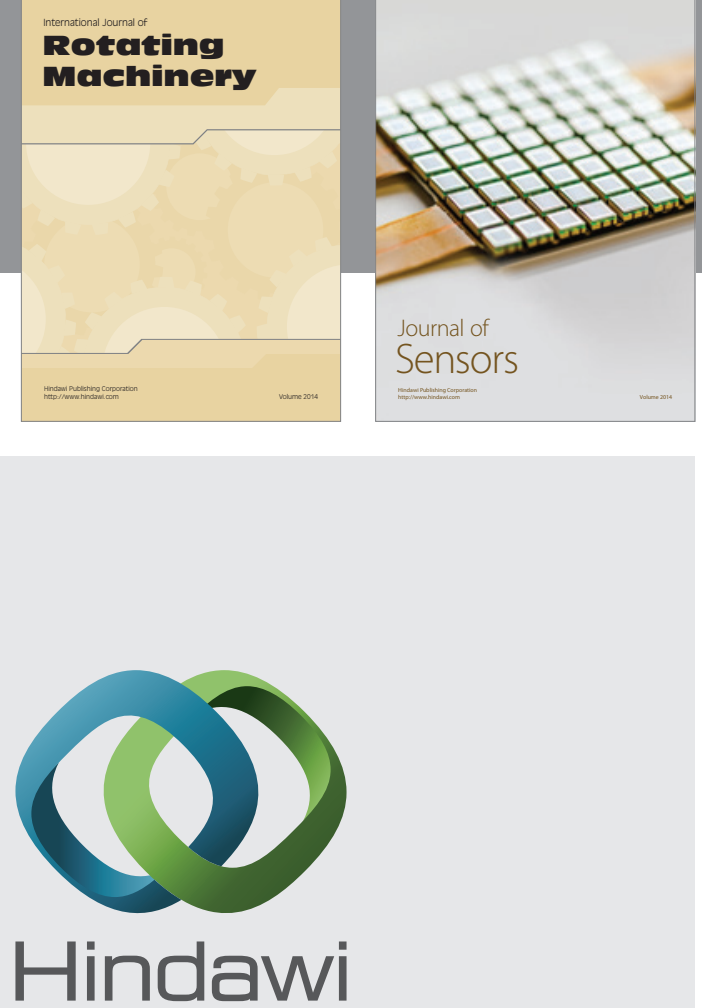

Submit your manuscripts at http://www.hindawi.com
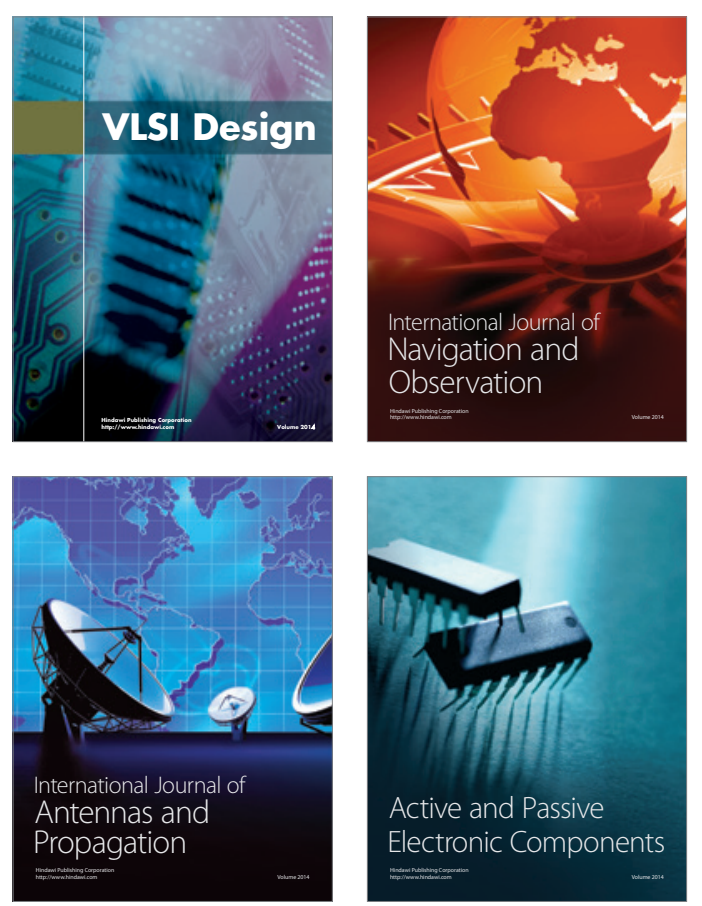
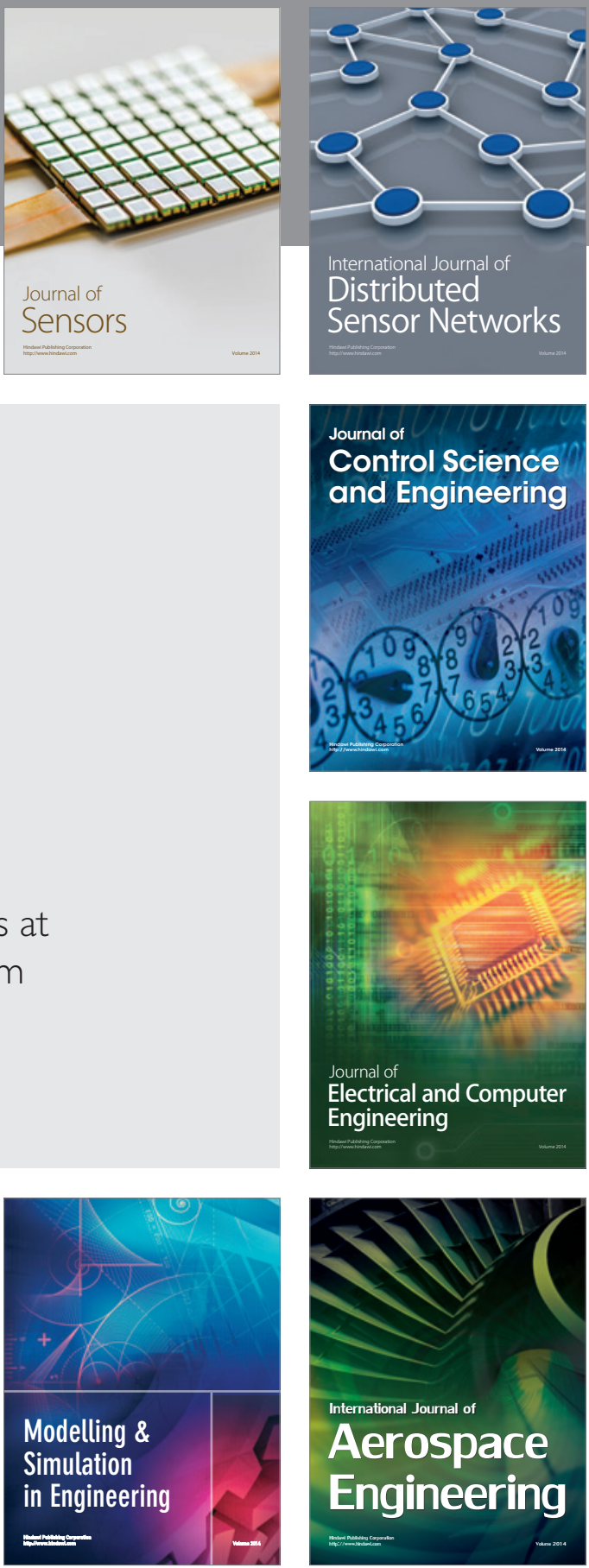

Journal of

Control Science

and Engineering
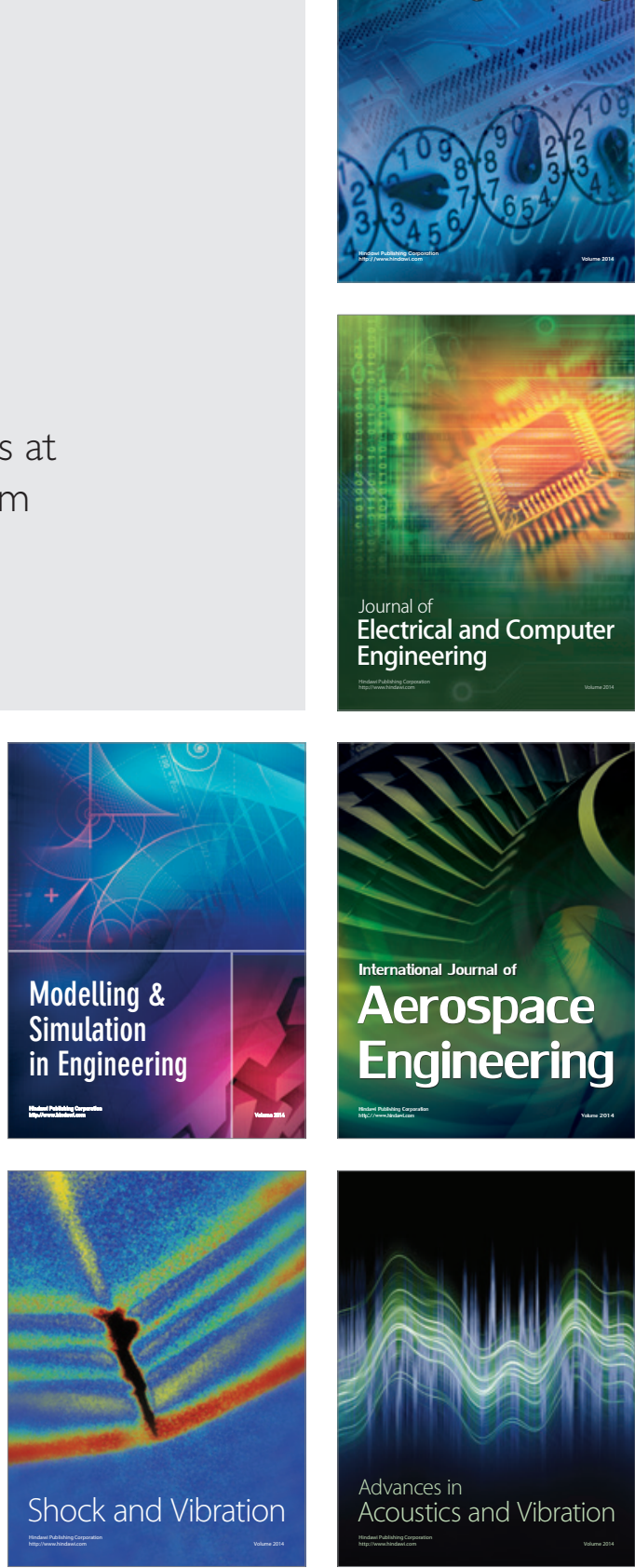